\title{
When does rheumatoid arthritis start and can it be stopped before it does?
}

\author{
Vivian P Bykerk, Johanna M Hazes²
}

Rheumatoid arthritis (RA) is characterised by a persistent and often destructive polyarticular synovitis involving large and small joints not infrequently associated with extra-articular features. This construct was classified by the American Rheumatism Association (ARA) 1987 classification criteria for RA. ${ }^{1}$ However, RA probably has a long prodrome with an asymptomatic phase followed by a period of unclassifiable musculoskeletal symptoms and signs before fully manifesting four out of seven of these criteria. Current concepts of pathogenesis presume that environmental triggers interact with genes such as those from the histocompatibility complex where an arthritogenic peptide triggers the innate immune response followed by upregulation of adaptive immunity. ${ }^{2}$ This would represent the preclinical phase of RA during which anti-citrullinated peptide antibodies (ACPAs) and then IgM-rheumatoid factor (IgM-RF) are generated. During this adaptive immune response, profound changes occur in the synovial membrane, leading to an influx of immune and inflammatory cells and increased vascularity of the joints followed by a destructive synovitis. ${ }^{2}$

Ultimately, patients develop a sufficient burden of synovitis that it is classifiable as RA. It is not easy to identify when RA actually starts clinically but it may be important to recognise preclinical RA at a much earlier time point in order to prevent progression to full-blown classifiable disease which becomes persistent and destructive. Starting from the presence of early arthritis or undifferentiated arthritis (UA) several prediction models to predict the development of RA-however, defined-have been derived. In all models the presence of

${ }^{1}$ Mount Sinai Hospital, University of Toronto, Toronto, Ontario, Canada

2Erasmus Medical Centre, University of Rotterdam, Rotterdam, The Netherlands

Correspondence to: Dr Vivian P Bykerk, Mount Sinai, 75 Francis Street, PBB-B3, Boston, MA 02115 , USA; vbykerk@partners.org
ACPAs is an independent strong predictor of the development of RA. ${ }^{3-6}$

Recently, researchers have attempted to identify patients in the prodromal phase during a period of very early inflammatory arthritis (VEIA) to determine if there are predictors that may indicate which patients go on to develop RA and if there is any treatment intervention that can prevent the inevitable progression to classifiable disease. These initiatives have been described in this issue of the journal. In the first article by Bos et al investigators attempted to determine if the presence of ACPAs or RF, or both, would be predictive of the development of synovitis in patients with arthralgias (see article on page 490). To examine the subject of early identification or prediction of persistent synovitis, Bos et al studied a cohort of patients presenting only with arthralgias, known to have either a positive RF or ACPA but who had no evidence of synovitis based on clinical examination. ${ }^{7}$ Twenty-nine of 147 of these patients developed synovitis over a median follow-up of 28 months. Patients were more likely to do so if they were ACPA positive than RF positive $(\mathrm{HR}=6.0 ; 95 \%$ CI 1.8 to $20.1 ; \mathrm{p}=0.003)$. From these data it could be inferred that RA is preceded by a prodromal phase of arthralgia.

These same authors went on to question, in a randomised controlled double blind trial, whether intramuscular dexamethasone given in two doses of $100 \mathrm{mg} 6$ weeks apart could reduce the presence of ACPAs and, presumably, prevent the onset of synovitis (see article on page 571). ${ }^{8}$ The rationale for studying this was that the presence of ACPAs have been found to be a dependent risk factor in the development of RA and thus reducing the presence of ACPAs may prevent the onset of synovitis in the continuum towards RA. However, despite finding that patients treated with dexamethasone had persistent reductions of ACPA and IgM-RF levels beginning after 1 month and continuing for 6 months, this reduction in ACPAs did not prevent the development of arthritis in these patients. ${ }^{8}$ This negative finding may be explained by the fact that this steroid regimen had too short a half-life to be of significant impact, that the study was not powered to answer this question or that patients already had synovitis that could have been measured by high-sensitivity imaging. No one has yet examined seropositive patients in the presymptomatic phase with high-sensitivity imaging at the phase of arthralgias to determine if there is measurable subclinical synovitis in these patients. Wakefield et al showed that $80 \%$ of patients presenting with monarthritis, when examined by musculoskeletal ultrasound were found to have synovitis in other joints. ${ }^{9}$ Also, Kraan et a ${ }^{10}$ showed that immunohistological features of synovitis precede clinically manifest arthritis in early RA. Thus it is possible that patients presenting in all these studies had more advanced joint disease than was clinically apparent. For this reason it is not entirely clear at what point to intervene to prevent full-blown classifiable RA.

Starting from the point where there is an identifiable synovitis, other investigators $^{11-13}$ go on to ask the question of whether there are ways of halting VEIA, even before it evolves to a classifiable form, perhaps even resetting the immune system and preventing the need for longterm treatment (see articles on pages 495, 503 and 510). Thinking along these lines, Verstappen et a ${ }^{12}$ sought to evaluate whether treating patients with VEIA (having a very early but still unclassified inflammatory polyarthritis) with a 3 -week course of intramuscular glucocorticoids (GCs), such as Depo-Medrone (DM), as compared with placebo could postpone the need for disease-modifying antirheumatic drugs (DMARDs) at 6 and 12 months and prevent the evolution into RA. Patients with VEIA of 4-10 weeks' duration were randomised to receive three injections of either $80 \mathrm{mg}$ intramuscular DM or placebo, given at weekly intervals. Subjects were assessed monthly for 6 months and then at 12 months. Twice as many patients receiving placebo as compared with those receiving intramuscular DM required DMARDs at 12 months. Only $10 \%$ in the treatment group as compared with $19.8 \%$ of patients in the placebo groups ever required DMARDs.

Machold et al ${ }^{11}$ also studied the effect of parenteral GC in patients with VEIA with the objective of determining if this intervention could induce drug-free 
clinical remission at weeks 12 and 52 . Subjects with $<16$ weeks of synovitis were randomised to receive either a single intramuscular injection of $120 \mathrm{mg}$ methylprednisolone or placebo and were followed up for 52 weeks. Unfortunately, there were no clinical benefits noted in the treatment arm after 2 weeks and the study was unable to show remission or delay in the development of RA by parenteral GC treatment. These data provide evidence for a beneficial but minimal effect of intramuscular GC in VEIA.

Previously, van Dongen et al ${ }^{14}$ examined this question in the PROMPT study (Probable rheumatoid arthritis: Methotrexate versus Placebo Treatment study) in which patients with undifferentiated inflammatory arthritis were randomised to receive methotrexate or placebo. They noted, particularly, that the majority of patients who were ACPA positive and given placebo went on to develop classifiable RA as defined by these 1987 ARA criteria. The implication of these research efforts was that patients who met these criteria are already in a later phase of the disease. Their findings also suggest that for some patients earlier treatment can prevent the disease or at least abolish the need for DMARDs.

To really prevent destructive disease when UA is already overt, a more powerful treatment strategy may be necessary. Emery et al ${ }^{13}$ sought to determine if a 6-month course of abatacept compared with placebo given to undifferentiated ACPA-positive subjects presenting with 8 months of early undifferentiated inflammatory arthritis would lower ACPA and RF levels and impact on radiographic progression. In this small, exploratory randomised controlled study, subjects treated with abatacept experienced lowering of their ACPA and RF titres, whereas those receiving placebo did not. Furthermore, there was worsening based on numerical differences in subjects receiving abatacept versus placebo both in radiographic scores and in osteitis scores (for those patients participating in the MRI substudy). The sample size was too small to know if this was statistically significant. Nonetheless, data from this study are consistent with those from Bos et $a l^{8}$ and Verstappen et al. ${ }^{12}$

Taken together, these data indicate that short-term, very early intervention in patients with as yet unclassifiable disease who are at risk for the development of classifiable RA may modify the course of the disease. What is not clear from these studies is whether these early limited treatment interventions: (a) prolong the development of true RA; (b) result in a less severe course or (c) completely prevent the development of the disease. In order to really understand the effects of early aggressive but short-term interventions all future studies should evaluate high-risk patients with VEIA or UA who have arthralgias and positive ACPAs. They should be designed, with adequate power, as randomised controlled trials with sufficient follow-up to determine if agents such as abatacept can truly prevent the development of RA as opposed to modifying or delaying the disease course.

Of interest, all evaluations have focused on the seropositive patient with unclassifiable disease and no studies evaluating these questions in seronegative patients have been carried out. Treating seronegative patients in the PROMPT study with methotrexate did not alter their disease course. ${ }^{14}$ The disease course in patients with seronegative disease may differ from that in patients with seropositive disease. More likely these patients represent a more heterogeneous population from the perspective of genetic predisposition and clinical disease course. Predictors of disease progression and persistence in this population still need to be identified. Once these are known, patients with a high likelihood of a destructive disease course would also need to be studied very early to determine which interventions might modify or prevent further disease.

Authors from all four of the VEIA studies discussed have considered the possibility that a single intervention can save patients from developing full-blown RA by intervening close to the start of the journey towards RA, but despite methodological variations in these trials only very early use of abatacept in an 'atrisk population' prevented progression of actual disease manifestations, in this case measured as joint destruction. There is a need to be able to identify RA close to its inception so that the disease course can be modified and, ideally, even halted or cured. It is still unclear as to how to best accomplish this. The use of GCs in patients with early inflammatory arthritis may delay the need for DMARDs but does not substantially alter the immune process to modify the course of the inflammatory process. However, although steroids do not change the nature of the disease, they may make it easier to control once classifiable RA has set in. But, to do so they must be given in high doses for a much longer period of time. ${ }^{15}$ However, the safety of this strategy has recently come in to question based on a meta-analysis. ${ }^{16}$ No study has looked at the effect of a longer period of high-dose oral GC in patients with VEIA. It may be that a combination of existing interventions will be required to accomplish the task of halting the disease at the stage of VEIA, or that a safe and cost effective intervention has yet to be discovered.

As future studies are designed to prevent RA it becomes important to identify RA when it starts. New classification criteria for RA will still identify patients with signs and symptoms but will not identify those with preclinical RA. VEIA, particularly at the preclinical phase, requires a more precise understanding of early immunological preclinical processes and will require a more sophisticated biomarker-based tool to identify it. It is hoped that these scientific analyses are being performed on biological samples from patients from these studies in order to learn how to stop a disease, which still becomes persistent and destructive for most patients and renders them dependent on drugs, often for life.

\section{Competing interests None.}

Provenance and peer review Commissioned; externally peer reviewed.

Accepted 12 January 2010

Ann Rheum Dis 2010;69:473-5.

doi:10.1136/ard.2009.116020

\section{REFERENCES}

1. Arnett FC, Edworthy SM, Bloch DA, et al. The American Rheumatism Association 1987 revised criteria for the classification of rheumatoid arthritis. Arthritis Rheum 1988;31:315-24.

2. Klareskog L, Catrina Al, Paget S. Rheumatoid arthritis. Lancet 2009;373:659-72.

3. Visser H, le Cessie S, Vos K, et al. How to diagnose rheumatoid arthritis early: a prediction model for persistent (erosive) arthritis. Arthritis Rheum 2002;46:357-65.

4. van der Helm-van Mil AH, le Cessie $\mathrm{S}$, van Dongen $\mathrm{H}$, et al. A prediction rule for disease outcome in patients with recent-onset undifferentiated arthritis. Arthritis Rheum 2007:56:433-40.

5. Nielen MM, van Schaardenburg D, Reesink HW, et al. Specific autoantibodies precede the symptoms of rheumatoid arthritis: a study of serial measurements in blood donors. Arthritis Rheum 2004;50:380-6.

6. Jørgensen KT, Wiik A, Pedersen M, et al. Cytokines, autoantibodies and viral antibodies in premorbid and postdiagnostic sera from patients with rheumatoid arthritis: case-control study nested in a cohort of Norwegian blood donors. Ann Rheum Dis 2008;67:860-6.

7. Bos WH, Wolbink GJ, Boers M, et al. Arthritis development in arthralgia patients is strongly associated with anti-citrullinated protein antibody status: a prospective cohort study. Ann Rheum Dis 2010;69:490-94.

8. Bos WH, Dijkmans BA, Boers M, et al. Effect of dexamethasone on autoantibody levels and arthritis development in arthralgia patients: a randomized trial. Ann Rheum Dis 2010;69:571-4. 
9. Wakefield RJ, Green MJ, Marzo-Ortega H, et al. Should oligoarthritis be reclassified? Ultrasound reveals a high prevalence of subclinical disease. Ann Rheum Dis 2004;63:382-5.

10. Kraan MC, Versendaal $H$, Jonker $M$, et al. Asymptomatic synovitis precedes clinically manifest arthritis. Arthritis Rheum 1998;41:1481-8.

11. Machold KP, RL, Smolen J J, Stamm TA, et al. The stop arthritis very early (SAVE) trial, an international multi-center, randomized, double-blind, placebo controlled trial on glucocorticoids in very early arthritis. Ann Rheum Dis;2010;69:495-502.
12. Verstappen SM, McCoy MJ, Roberts C, et al. The beneficial effects of a 3 week course of intramuscular glucocorticoid injections in patients with very early inflammatory polyarthritis: Results of the STIVEA trial. Ann Rheum Dis 2010;69:503-9.

13. Emery P, Durez P, Dougados M, et al. The impact of T-cell co-stimulation modulation in patients with undifferentiated inflammatory arthritis or very early rheumatoid arthritis: a clinical and imaging study of abatacept. Ann Rheum Dis 2010;69:510-16.

14. van Dongen $\mathbf{H}$, van Aken J, Lard $L R$, et al. Efficacy of methotrexate treatment in patients with probable rheumatoid arthritis: a double-blind, randomized, placebo-controlled trial. Arthritis Rheum 2007:56:1424-32.

15. Goekoop-Ruiterman YP, de Vries-Bouwstra JK, Allaart CF, et al. Clinical and radiographic outcomes of four different treatment strategies in patients with early rheumatoid arthritis (the BeSt study): a randomized, controlled trial. Arthritis Rheum 2005;52:3381-90.

16. Hoes JN, Jacobs JW, Verstappen SM, et al Adverse events of low- to medium-dose oral glucocorticoids in inflammatory diseases: a metaanalysis. Ann Rheum Dis 2009;68:1833-8. 